\title{
Prevalence and molecular epidemiology of methicillin-resistant Staphylococcus aureus colonization with and without methicillin-resistant coagulase-negative Staphylococci in HIV-infected patients
}

\section{Ying Li}

Guangdong Pharmaceutical University

Jialing Lin

University of New South Wales

Linghua Li

Guangzhou Eighth People's Hospital

\section{Weiping Cai}

Guangzhou Eighth People's Hospital

\section{Yingying Wang}

Guangdong Pharmaceutical University

Jiaping Ye

Beihai People's Hospital

Suiping He

Guangdong Pharmaceutical University

\section{Wencui Zhang}

Guangdong Pharmaceutical University

Xiaohua Ye

Guangdong Pharmaceutical University

Zhenjiang Yao ( $\square$ zhjyao2001@yahoo.com )

https://orcid.org/0000-0002-2156-7896

Research article

Keywords: Methicillin-resistant Staphylococcus aureus, Methicillin-resistant coagulase-negative Staphylococci, Colonization, HIV

Posted Date: March 17th, 2020

DOl: https://doi.org/10.21203/rs.3.rs-17504/v1 
License: (c) (i) This work is licensed under a Creative Commons Attribution 4.0 International License. Read Full License 


\section{Abstract}

\section{Background}

The global prevalence of methicillin-resistant Staphylococcus aureus (MRSA) colonization in human immunodeficiency virus (HIV)-infected patients is increasing, but data about it was limited in mainland China. This study aims to investigate the epidemiology of MRSA nasal colonization among HIV-infected patients in mainland China and also evaluate the impact of methicillin-resistant coagulase-negative Staphylococci (MRCoNS).

\section{Methods}

This cross-sectional study was designed to collect nasal samples and individual information for HIVinfected participants. Risk factors and phenotypic and molecular characteristics among those with MRSA colonization comparing those with and without MRCoNS co-colonization were analyzed.

\section{Results}

We found $119(11.89 \%)$ out of 1001 HIV-infected patients were colonized with MRSA, including 41 $(4.10 \%)$ with MRCoNS and $78(7.79 \%)$ without MRCoNS. Having a history of respiratory tract infection in the previous 6 months(adjusted OR=1.64, 95\% Cl: 1.04-2.57) and male gender (aOR=3.92, 95\% Cl: $1.20-$ 12.85) were risk factors for the overall MRSA and MRSA without MRCoNS colonization, respectively. No risk factor was associated with co-colonization of MRSA and MRCoNS. The proportions of antibiotic resistance and toxin genes as well as the distribution of molecular types for MRSA isolates were equivalent between subjects with and without MRCoNS isolates (P-value $\geq 0.05$ ).

\section{Conclusions}

HIV-infected patients remain a highly vulnerable population for MRSA colonization, and men and prior history of respiratory tract infection are risk factors. Risk factors and phenotypic and molecular characteristics of MRSA colonization were not affected by co-colonizing with MRCoNS.

\section{Background}

Methicillin resistance in Staphylococcus is a serious public health concern as it compromises first-line drugs for treating staphylococcal infections in humans [1]. As a common cause of severe infections, methicillin-resistant Staphylococcus aureus (MRSA) is still widespread in both health facilities and the community [2, 3]. People with infections caused by MRSA are at increased risk of worse clinical outcomes and death as well as consume more health-care resources than people with a non-resistant form of the infection $[4,5]$.

Coagulase-negative Staphylococci (CoNS) are commonly found in food, animals,environment and humans [6]. They not only can cause severe animal diseases, but also are one of the leading etiologic 
agents for nosocomial bloodstream infection in humans, especially immunocompromised patients. Studies suggested methicillin-resistant CoNS (MRCoNS) as a source of mecA, the methicillin resistance gene, and has the potential contribution to the emergence of MRSA [7]. Studies also reported the horizontal cross-propagation of resistance genes could lead to the co-colonization of MRSA and MRCoNS in humans [8]. In other words, MRCoNS might affect the risk of MRSA colonization.

Study have shown that the global and regional prevalence of MRSA colonization in HIV-infected patients increasing[9]. Moreover, the epidemiology of MRSA in HIV-infected patients have been explored in Taiwan, China[10-11]. But data on the prevalence and molecular characteristics of MRSA isolated among HIVinfected patients was limited in mainland China. we thus aimed to investigate the prevalence and molecular epidemiology of MRSA colonization among this vulnerable population in mainland China and also examine the impact of MRCoNS by risk factors as well as phenotypic, genotypic and molecular characteristics on MRSA colonization.

\section{Methods}

\section{Study design and population}

HIV-infected participants were enrolled from a large public HIV clinic with a population of more than 9000 of HIV-infected patients, about $90 \%$ of all HIV-infected patients in Guangzhou city, that is affiliated with the Guangzhou Eighth People's Hospital. Patients in the clinic were eligible for enrollment if they were $\geq$ 18 years of age and agreed with participation. The study was approved by the Ethics Committee of Guangdong Pharmaceutical University. All participants signed an informed consent.

\section{Data collection}

Nasal cultures were obtained from enrolled participants, and a face-to-face questionnaire was administered. The questionnaire included demographics (age, gender, martial status and education level), behavior-related information (smoking status, number of people living in the household, use of shared items and living with mammalian pets), details of community-based risk factors (location and type of residence, sexual behavior, street drug abuse and incarceration), medical information (hospitalization, surgeries, skin infection and respiratory tract infection) and HIV-related information (CD4 count, viral load, trimethoprim-sulfamethoxazole prophylaxis, antiretroviral therapy, antifungal medication and tuberculosis medication). Nasal swabs were obtained by swabbing both nares of participants for further experiments.

\section{Laboratory processing}

Swabs were inoculated into $7.5 \% \mathrm{NaCl}$ broth and incubated overnight at $37^{\circ} \mathrm{C}$ for enrichment. Isolates were identified as Staphylococci if they were positive for chromogenic reaction in sodium chloride mannitol medium, Gram staining, catalase test and hemolysis test. All Staphylococci isolates were then classified into Staphylococcus aureus (S. aureus) and CoNS by the coagulase test. Both S. aureus and CoNS isolates were tested for antibiotic resistance by using Kirby-Bauer disk diffusion method based on 
the 2017 Clinical Laboratory Standards Institute guidelines. Twelve antibiotics were tested, including cefoxitin, penicillin, erythromycin, clindamycin, tetracycline, rifampicin, macrodantin, moxifloxacin, trimethoprim-sulfamethoxazole, teicoplanin, linezolid and gentamicin. Isolates resistant to cefoxitin were confirmed as methicillin resistant. Genotypic analysis with pulsed-field gel electrophoresis was performed on all confirmed MRSA isolates, including the Panton-Valentine leukocidin gene (pvl), two exfoliating genes (eta and etb) and the toxic shock syndrome toxin gene (tst) [12]. Staphylococcal cassette chromosome mec (SCCmec) typing [13] and multilocus sequence typing (MLST) [14] for all MRSA isolates were also analyzed.

\section{Statistical analysis}

Frequencies and proportions were calculated to describe the characteristics of HIV-infected patients by MRSA colonization. Risk factors for MRSA colonization was examined by using logistics regression analysis. Significant factors in univariate analysis were selected into multivariate models. Differences of phenotypic and genotypic characteristics for MRSA isolates between with and without MRCoNS were examined by Pearson Chi-square test or Fisher's exact test. Two-sided P-values $<0.05$ were considered as statistically significant. All statistical analyses were performed using Stata v15.1 (Collage Station, TX). The sequence type relationship of MRSA isolates between with and without MRCoNS isolates was visualized using Phyloviz 2.0 (http://www.phyloviz.net/).

\section{Results}

\section{Participant characteristics}

A total of $1001 \mathrm{HIV}$-infected patients were eligible and enrolled. We found 119 (11.89\%) patients were with MRSA colonization, including 41 (4.10\%) with MRCoNS and 78 (7.79\%) without MRCoNS (Fig. 1). The participant characteristics for MRSA colonization are shown in Table 1. The mean age was 37.24 (SD, 11.35 ) years, and $84.42 \%$ of the study population was male. Most patients $(91.41 \%)$ had antiretroviral therapy. The median and interquartile range of CD 4 count was $330(500-1022) \mathrm{cells} / \mathrm{mL}$, and the median and interquartile range of viral load was 20 (20-1630000) copies $/ \mathrm{mL}$. Only $6.6 \%$ and $5.9 \%$ of our patients had antifungal and tuberculosis medication by self-report. The proportions of diagnosis with skin and respiratory tract infections in the previous 6 months were $44.58 \%$ and $67.47 \%$. 
Table 1

Characteristics of HIV-infected patients by MRSA colonization

\begin{tabular}{|c|c|c|c|c|}
\hline Characteristics & $\begin{array}{l}\text { Overall } \\
(\mathrm{N}= \\
1001)\end{array}$ & $\begin{array}{l}\text { MRSA } \\
(\mathrm{N}= \\
119)\end{array}$ & $\begin{array}{l}\text { MRSA with } \\
\text { MRCoNS } \\
(\mathrm{N}=41)\end{array}$ & $\begin{array}{l}\text { MRSA without } \\
\text { MRCoNS } \\
(\mathrm{N}=78)\end{array}$ \\
\hline \multicolumn{5}{|l|}{ Gender } \\
\hline Male & 845 & $\begin{array}{l}107 \\
(12.66)\end{array}$ & $33(3.91)$ & $74(8.76)$ \\
\hline Female & 156 & $\begin{array}{l}12 \\
(7.69)\end{array}$ & $8(5.13)$ & $4(2.56)$ \\
\hline \multicolumn{5}{|l|}{ Age, year } \\
\hline$<30$ & 326 & $\begin{array}{l}45 \\
(13.80)\end{array}$ & $17(5.21)$ & $28(8.59)$ \\
\hline $30-45$ & 426 & $\begin{array}{l}48 \\
(11.27)\end{array}$ & $12(2.82)$ & $36(8.45)$ \\
\hline$\geq 45$ & 247 & $\begin{array}{l}26 \\
(10.53)\end{array}$ & $12(4.86)$ & $14(5.67)$ \\
\hline \multicolumn{5}{|l|}{ Marital status } \\
\hline Never married & 464 & $\begin{array}{l}67 \\
(14.44)\end{array}$ & $23(4.96)$ & $44(9.48)$ \\
\hline Married & 449 & $\begin{array}{l}45 \\
(10.02)\end{array}$ & $15(3.34)$ & $30(6.68)$ \\
\hline Divorced or widowed & 84 & $\begin{array}{l}7 \\
(8.33)\end{array}$ & $3(3.57)$ & $4(4.76)$ \\
\hline Others & 2 & $\begin{array}{l}0 \\
(0.00)\end{array}$ & $0(0.00)$ & $0(0.00)$ \\
\hline \multicolumn{5}{|l|}{ Education } \\
\hline Primary school or below & 102 & $\begin{array}{l}9 \\
(8.82)\end{array}$ & $6(5.88)$ & $3(2.94)$ \\
\hline Middle school & 302 & $\begin{array}{l}33 \\
(10.97)\end{array}$ & $11(3.64)$ & $22(7.28)$ \\
\hline High school & 233 & $\begin{array}{l}24 \\
(10.30)\end{array}$ & $10(4.29)$ & $14(6.01)$ \\
\hline
\end{tabular}

MRSA, methicillin-resistant Staphylococcus aureus; MRCoNS, methicillin-resistant coagulase-negative Staphylococci.

* Within previous 6 months. 


\begin{tabular}{|c|c|c|c|c|}
\hline Characteristics & $\begin{array}{l}\text { Overall } \\
(\mathrm{N}= \\
1001)\end{array}$ & $\begin{array}{l}\text { MRSA } \\
(\mathrm{N}= \\
119)\end{array}$ & $\begin{array}{l}\text { MRSA with } \\
\text { MRCoNS } \\
(\mathrm{N}=41)\end{array}$ & $\begin{array}{l}\text { MRSA without } \\
\text { MRCoNS } \\
(\mathrm{N}=78)\end{array}$ \\
\hline College degree or above & 364 & $\begin{array}{l}53 \\
(14.56)\end{array}$ & $14(3.85)$ & $39(10.71)$ \\
\hline \multicolumn{5}{|l|}{ Been arrested } \\
\hline No & 947 & $\begin{array}{l}110 \\
(11.63)\end{array}$ & $36(3.80)$ & $74(7.81)$ \\
\hline Yes & 54 & $\begin{array}{l}9 \\
(16.67)\end{array}$ & $5(9.26)$ & $4(7.41)$ \\
\hline \multicolumn{5}{|l|}{ Current smoker } \\
\hline No & 638 & $\begin{array}{l}86 \\
(13.48)\end{array}$ & $30(4.69)$ & $56(8.78)$ \\
\hline Yes & 362 & $\begin{array}{l}33 \\
(9.12)\end{array}$ & $11(3.04)$ & $22(6.08)$ \\
\hline \multicolumn{5}{|l|}{ Street drug abuse* } \\
\hline No & 932 & $\begin{array}{l}108 \\
(11.59)\end{array}$ & $36(3.86)$ & $72(7.73)$ \\
\hline Yes & 55 & $\begin{array}{l}9 \\
(16.36)\end{array}$ & $4(7.27)$ & $5(9.09)$ \\
\hline \multicolumn{5}{|c|}{$\begin{array}{l}\text { Number of people living in the household } \\
(\geq 5 \text { members })^{\star}\end{array}$} \\
\hline No & 878 & $\begin{array}{l}105 \\
(11.96)\end{array}$ & 35 (3.99) & $70(7.97)$ \\
\hline Yes & 120 & $\begin{array}{l}13 \\
(10.83)\end{array}$ & $5(4.17)$ & $8(6.67)$ \\
\hline \multicolumn{5}{|c|}{ Use of shared household items* } \\
\hline No & 930 & $\begin{array}{l}112 \\
(12.04)\end{array}$ & $39(4.19)$ & $73(7.85)$ \\
\hline Yes & 70 & $\begin{array}{l}7 \\
(10.00)\end{array}$ & $2(2.86)$ & $5(7.14)$ \\
\hline
\end{tabular}

MRSA, methicillin-resistant Staphylococcus aureus; MRCoNS, methicillin-resistant coagulase-negative Staphylococci.

* Within previous 6 months. 


\begin{tabular}{|c|c|c|c|c|}
\hline Characteristics & $\begin{array}{l}\text { Overall } \\
(\mathrm{N}= \\
1001)\end{array}$ & $\begin{array}{l}\text { MRSA } \\
(\mathrm{N}= \\
119)\end{array}$ & $\begin{array}{l}\text { MRSA with } \\
\text { MRCoNS } \\
(\mathrm{N}=41)\end{array}$ & $\begin{array}{l}\text { MRSA without } \\
\text { MRCoNS } \\
(\mathrm{N}=78)\end{array}$ \\
\hline No & 765 & $\begin{array}{l}90 \\
(11.76)\end{array}$ & $32(4.18)$ & $58(7.58)$ \\
\hline Yes & 229 & $\begin{array}{l}28 \\
(12.23)\end{array}$ & $8(3.49)$ & $20(8.73)$ \\
\hline \multicolumn{5}{|l|}{ Hospitalization* } \\
\hline No & 826 & $\begin{array}{l}105 \\
(12.71)\end{array}$ & $37(4.46)$ & $68(8.23)$ \\
\hline Yes & 171 & $\begin{array}{l}14 \\
(8.19)\end{array}$ & $4(2.34)$ & $10(5.85)$ \\
\hline \multicolumn{5}{|l|}{ Surgeries* } \\
\hline No & 882 & $\begin{array}{l}107 \\
(12.13)\end{array}$ & $37(4.16)$ & $70(7.94)$ \\
\hline Yes & 111 & $\begin{array}{l}11 \\
(9.91)\end{array}$ & $4(3.60)$ & $7(6.31)$ \\
\hline \multicolumn{5}{|c|}{ Skin and soft-tissue infection* } \\
\hline No & 557 & $\begin{array}{l}61 \\
(10.95)\end{array}$ & $25(4.49)$ & $36(6.46)$ \\
\hline Yes & 444 & $\begin{array}{l}58 \\
(13.06)\end{array}$ & $16(3.60)$ & $42(9.46)$ \\
\hline \multicolumn{5}{|c|}{ Respiratory tract infection* } \\
\hline No & 325 & $\begin{array}{l}28 \\
(8.62)\end{array}$ & $11(3.38)$ & $17(5.23)$ \\
\hline Yes & 674 & $\begin{array}{l}91 \\
(13.50)\end{array}$ & $30(4.45)$ & $61(9.05)$ \\
\hline \multicolumn{5}{|l|}{ Antibiotic use ${ }^{\star}$} \\
\hline No & 536 & $\begin{array}{l}57 \\
(10.63)\end{array}$ & $26(4.72)$ & $31(5.78)$ \\
\hline Yes & 450 & $\begin{array}{l}60 \\
(13.33)\end{array}$ & 15 (3.33) & $45(10.00)$ \\
\hline
\end{tabular}

MRSA, methicillin-resistant Staphylococcus aureus; MRCoNS, methicillin-resistant coagulase-negative Staphylococci.

* Within previous 6 months. 


\begin{tabular}{|c|c|c|c|c|}
\hline Characteristics & $\begin{array}{l}\text { Overall } \\
(\mathrm{N}= \\
1001)\end{array}$ & $\begin{array}{l}\text { MRSA } \\
(\mathrm{N}= \\
119)\end{array}$ & $\begin{array}{l}\text { MRSA with } \\
\text { MRCoNS } \\
(\mathrm{N}=41)\end{array}$ & $\begin{array}{l}\text { MRSA without } \\
\text { MRCoNS } \\
(\mathrm{N}=78)\end{array}$ \\
\hline \multicolumn{5}{|c|}{$\begin{array}{l}\text { Trimethoprim-sulfamethoxazole } \\
\text { prophylaxis* }\end{array}$} \\
\hline No & 771 & $\begin{array}{l}94 \\
(12.19)\end{array}$ & $31(4.02)$ & $63(8.17)$ \\
\hline Yes & 206 & $\begin{array}{l}23 \\
(11.17)\end{array}$ & $9(4.37)$ & $14(6.80)$ \\
\hline \multicolumn{5}{|c|}{ Antifungal medication* } \\
\hline No & 918 & $\begin{array}{l}113 \\
(12.31)\end{array}$ & $39(4.25)$ & $74(8.06)$ \\
\hline Yes & 66 & $\begin{array}{l}4 \\
(6.06)\end{array}$ & $1(1.52)$ & $3(4.55)$ \\
\hline \multicolumn{5}{|c|}{ Tuberculosis medication* } \\
\hline No & 929 & $\begin{array}{l}111 \\
(11.95)\end{array}$ & $39(4.20)$ & $72(7.75)$ \\
\hline Yes & 59 & $\begin{array}{l}7 \\
(11.86)\end{array}$ & $1(1.69)$ & $6(10.17)$ \\
\hline \multicolumn{5}{|c|}{ HIV transmission (sexual) } \\
\hline No & 326 & $\begin{array}{l}32 \\
(9.82)\end{array}$ & $11(3.37)$ & $21(6.44)$ \\
\hline Yes & 672 & $\begin{array}{l}86 \\
(12.80)\end{array}$ & $29(3.37)$ & $57(8.48)$ \\
\hline \multicolumn{5}{|l|}{ HIV medication } \\
\hline No & 86 & $\begin{array}{l}16 \\
(18.60)\end{array}$ & $6(6.98)$ & $10(11.63)$ \\
\hline Yes & 911 & $\begin{array}{l}103 \\
(11.30)\end{array}$ & $35(3.83)$ & $68(7.46)$ \\
\hline \multicolumn{5}{|l|}{ CD4 count } \\
\hline$<200$ & 283 & $\begin{array}{l}35 \\
(12.28)\end{array}$ & $12(4.24)$ & $23(8.13)$ \\
\hline
\end{tabular}

MRSA, methicillin-resistant Staphylococcus aureus; MRCoNS, methicillin-resistant coagulase-negative Staphylococci.

* Within previous 6 months. 


\begin{tabular}{|c|c|c|c|c|}
\hline Characteristics & $\begin{array}{l}\text { Overall } \\
(\mathrm{N}= \\
1001)\end{array}$ & $\begin{array}{l}\text { MRSA } \\
(\mathrm{N}= \\
119)\end{array}$ & $\begin{array}{l}\text { MRSA with } \\
\text { MRCoNS } \\
(\mathrm{N}=41)\end{array}$ & $\begin{array}{l}\text { MRSA without } \\
\text { MRCoNS } \\
(\mathrm{N}=78)\end{array}$ \\
\hline $200-500$ & 462 & $\begin{array}{l}62 \\
(13.60)\end{array}$ & $18(3.90)$ & $44(9.52)$ \\
\hline$\geq 500$ & 248 & $\begin{array}{l}22 \\
(8.46)\end{array}$ & $9(3.63)$ & $10(4.03)$ \\
\hline \multicolumn{5}{|c|}{ Viral load, copies/mL } \\
\hline Undetected $(<50)$ & 565 & $\begin{array}{l}67 \\
(11.86)\end{array}$ & $22(3.89)$ & $45(7.96)$ \\
\hline Detected $(\geq 50)$ & 82 & $\begin{array}{l}10 \\
(12.20)\end{array}$ & $4(4.88)$ & $6(7.32)$ \\
\hline \multicolumn{5}{|c|}{$\begin{array}{l}\text { MRSA, methicillin-resistant Staphylococcus aureus; MRCoNS, methicillin-resistant coagulase-negativ } \\
\text { Staphylococci. }\end{array}$} \\
\hline
\end{tabular}

\section{Risk factors}

Significant factors associated with MRSA colonization on univariate analysis included high education level (odds ratio [OR] $=1.47,95 \%$ confidence interval [Cl]: 1.00-2.17) and diagnosis of respiratory tract infection in the previous 6 months ( $\mathrm{OR}=1.66,95 \% \mathrm{Cl}$ : 1.06-2.59) (Table 2). Four risk factors, including male gender ( $\mathrm{OR}=3.61,95 \% \mathrm{Cl}: 1.30-10.03)$, high education level $(\mathrm{OR}=1.84,95 \% \mathrm{Cl}: 1.15-2.92)$, diagnosis of respiratory tract infection in the previous 6 months $(\mathrm{OR}=1.83,95 \% \mathrm{Cl}: 1.05-3.19)$ and prior history of antibiotic use (OR $=1.78,95 \% \mathrm{Cl}: 1.11-2.87)$ were found for MRSA without MRCoNS colonization. However, no significant risk factor for co-colonization of MRSA and MRCoNS was found.

Using a multivariate logistic regression model accounting for gender, age and significant factors on univariate analysis (including martial status, education level, smoking status and HIV medication), diagnosis of respiratory tract infection in the previous 6 months (adjusted OR [aOR] $=1.64,95 \% \mathrm{Cl}$ : 1.042.57) remained a risk factor for MRSA colonization. For MRSA without MRCoNS colonization, male gender (aOR $=3.92,95 \% \mathrm{Cl}: 1.20-12.85)$ remains to be a risk factor after adjusting for age, education level, diagnosis of respiratory tract infection in the previous 6 months as well as antibiotic use. 
Table 2

Univariate analyses of risk factors associated with MRSA colonization in HIV-infected patients [OR (95\% $\mathrm{Cl})]$

\begin{tabular}{|c|c|c|c|}
\hline Characteristics & $\begin{array}{l}\text { MRSA } \\
(\mathrm{N}=119)\end{array}$ & $\begin{array}{l}\text { MRSA with } \\
\text { MRCoNS } \\
(\mathrm{N}=41)\end{array}$ & $\begin{array}{l}\text { MRSA without } \\
\text { MRCoNS } \\
(\mathrm{N}=78)\end{array}$ \\
\hline Gender (male) & $\begin{array}{l}1.74(0.93- \\
3.24)\end{array}$ & $0.80(0.36-1.78)$ & $3.61(1.30-10.03)$ \\
\hline Age ( $\geq 45$ years $)$ & $\begin{array}{l}0.73(0.44- \\
1.22)\end{array}$ & $1.23(0.62-2.46)$ & $0.65(0.36-1.19)$ \\
\hline Marital status (married) & $\begin{array}{l}0.66(0.44- \\
0.99)\end{array}$ & $0.68(0.36-1.30)$ & $0.74(0.46-1.18)$ \\
\hline Education (college degree or above) & $\begin{array}{l}1.47(1.00- \\
2.17)\end{array}$ & $0.95(0.49-1.84)$ & $1.84(1.15-2.92)$ \\
\hline Current smoker & $\begin{array}{l}0.64(0.42- \\
0.98)\end{array}$ & $0.62(0.30-1.24)$ & $0.66(0.40-1.10)$ \\
\hline Use of shared household items* & $\begin{array}{l}0.81(0.36- \\
1.82)\end{array}$ & $0.67(0.16-2.82)$ & $0.89(0.35-2.28)$ \\
\hline Living with mammalian pets & $\begin{array}{l}1.04(0.66- \\
1.64)\end{array}$ & $0.84(0.38-1.85)$ & $1.16(0.68-1.97)$ \\
\hline Hospitalization* & $\begin{array}{l}0.61(0.34- \\
1.10)\end{array}$ & $0.50(0.17-1.41)$ & $0.68(0.34-1.34)$ \\
\hline Surgeries* & $\begin{array}{l}0.80(0.41- \\
1.53)\end{array}$ & $0.84(0.29-2.40)$ & $0.78(0.35-1.73)$ \\
\hline Respiratory tract infection* & $\begin{array}{l}1.66(1.06- \\
2.59)\end{array}$ & $1.39(0.69-2.81)$ & $1.83(1.05-3.19)$ \\
\hline Antibiotic use* & $\begin{array}{l}1.29(0.88- \\
1.90)\end{array}$ & $0.71(0.37-1.36)$ & $1.78(1.11-2.87)$ \\
\hline Antifungal medication* & $\begin{array}{l}0.46(0.16- \\
1.29)\end{array}$ & $0.33(0.04-2.46)$ & $0.53(0.16-1.72)$ \\
\hline $\begin{array}{l}\text { Trimethoprim-sulfamethoxazole } \\
\text { prophylaxis* }\end{array}$ & $\begin{array}{l}0.91(0.56- \\
1.47)\end{array}$ & $1.07(0.50-2.30)$ & $0.82(0.45-1.50)$ \\
\hline HIV medication & $\begin{array}{l}0.56(0.31- \\
1.00)\end{array}$ & $0.51(0.21-1.24)$ & $0.59(0.29-1.20)$ \\
\hline CD4 count $(\geq 200)$ & $\begin{array}{l}0.91(0.60- \\
1.39)^{(0)}\end{array}$ & $0.89(0.44-1.78)$ & $0.93(0.56-1.54)$ \\
\hline
\end{tabular}

OR, odds ratio; $\mathrm{Cl}$, confidence interval; MRSA, methicillin-resistant Staphylococcus aureus; MRCoNS, methicillin-resistant coagulase-negative Staphylococci. * Within previous 6 months. 


\begin{tabular}{|llll|}
\hline Characteristics & $\begin{array}{l}\text { MRSA } \\
(\mathbf{N = 1 1 9 )}\end{array}$ & $\begin{array}{l}\text { MRSA with } \\
\text { MRCoNS } \\
\mathbf{N}=\mathbf{4 1})\end{array}$ & $\begin{array}{l}\text { MRSA without } \\
\text { MRCoNS } \\
\mathbf{( N = 7 8 )}\end{array}$ \\
\hline Viral load ( $\geq 50$ copies $/ \mathrm{mL})$ & $\begin{array}{l}1.26(0.42-3.75) \\
\text { 2.10) }\end{array}$ & $0.92(0.38-2.24)$ \\
\hline $\begin{array}{l}\text { OR, odds ratio; Cl, confidence interval; MRSA, methicillin-resistant Staphylococcus aureus; MRCoNS, } \\
\text { methicillin-resistant coagulase-negative Staphylococci. * Within previous 6 months. }\end{array}$ \\
\hline
\end{tabular}

\section{Phenotypic characteristics}

Antibiotic susceptibility testing was carried out on the 119 MRSA isolates. One hundred and six (89.08\%) were resistant to penicillin, $59(49.58 \%)$ to erythromycin, $34(28.57 \%)$ to clindamycin, $32(26.89 \%)$ to tetracycline, $21(17.65 \%)$ to rifampicin, $20(16.81 \%)$ to macrodantin, $18(15.13 \%)$ to moxifloxacin, 17 (14.29\%) to trimethoprim-sulfamethoxazole, $12(10.08 \%)$ to teicoplanin, $10(8.40 \%)$ to linezolid, and 6 $(5.04 \%)$ to gentamicin. The proportions of antibiotic resistance were no difference for MRSA isolates between with and without MRCoNS (Table 3). 
Table 3

Phenotypic and genotypic characteristics of MRSA isolates in HIV-infected patients

\begin{tabular}{|c|c|c|c|c|c|}
\hline Characteristics & $\begin{array}{l}\text { Overall } \\
(N=119)\end{array}$ & $\begin{array}{l}\text { MRSA with } \\
\text { MRCoNS } \\
(\mathrm{N}=41)\end{array}$ & $\begin{array}{l}\text { MRSA without } \\
\text { MRCoNS } \\
(\mathrm{N}=78)\end{array}$ & $x^{2}$ & $\begin{array}{l}\text { P- } \\
\text { value }\end{array}$ \\
\hline \multicolumn{6}{|l|}{$\begin{array}{l}\text { Phenotypes (non- } \\
\text { susceptive) }\end{array}$} \\
\hline Penicillin & $\begin{array}{l}106 \\
(89.08)\end{array}$ & $39(95.12)$ & $67(85.90)$ & 2.35 & 0.125 \\
\hline Erythromycin & $\begin{array}{l}59 \\
(49.58)\end{array}$ & $25(60.98)$ & $48(61.54)$ & 0.004 & 0.952 \\
\hline Clindamycin & $\begin{array}{l}34 \\
(28.57)\end{array}$ & $20(48.78)$ & $30(38.46)$ & 1.17 & 0.278 \\
\hline Tetracycline & $\begin{array}{l}32 \\
(26.89)\end{array}$ & $17(41.46)$ & $24(30.77)$ & 1.36 & 0.243 \\
\hline Rifampicin & $\begin{array}{l}21 \\
(17.65)\end{array}$ & $11(26.83)$ & $12(15.38)$ & 2.26 & 0.133 \\
\hline Macrodantin & $\begin{array}{l}20 \\
(16.81)\end{array}$ & $15(36.59)$ & $23(29.49)$ & 0.62 & 0.430 \\
\hline Moxifloxacin & $\begin{array}{l}18 \\
(15.13)\end{array}$ & 10 (24.39) & $18(23.08)$ & 0.02 & 0.872 \\
\hline $\begin{array}{l}\text { Trimethoprim- } \\
\text { sulfamethoxazole }\end{array}$ & $\begin{array}{l}17 \\
(14.29)\end{array}$ & $7(17.07)$ & $17(21.79)$ & 0.37 & 0.542 \\
\hline Teicoplanin & $\begin{array}{l}12 \\
(10.08)\end{array}$ & $16(39.02)$ & $23(29.49)$ & 1.11 & 0.292 \\
\hline Linezolid & $10(8.40)$ & $5(12.20)$ & $5(6.41)$ & 1.17 & 0.280 \\
\hline Gentamicin & $6(5.04)$ & $3(7.32)$ & $4(5.13)$ & 0.23 & 0.630 \\
\hline \multicolumn{6}{|l|}{ Genotypes (positive) } \\
\hline $\mathrm{pvl}$ & $5(4.20)$ & $1(2.44)$ & $4(5.13)$ & NA & $0.436 *$ \\
\hline eta & $0(0.00)$ & $0(0.00)$ & $0(0.00)$ & NA & NA \\
\hline etb & $0(0.00)$ & $0(0.00)$ & $0(0.00)$ & NA & NA \\
\hline tst & $5(4.20)$ & $0(0.00)$ & $5(6.41)$ & NA & $0.116 *$ \\
\hline
\end{tabular}




\section{Molecular characteristics}

The results of toxin genes showed pvl and tst genes were respectively identified in 5 (4.20\%) and 5 (4.20\%) MRSA isolates, no isolate carrying eta or etb genes. Indifference distribution of toxin genes was found for MRSA isolates between with and without MRCoNS (Table 3). For the results of SCCmec typing,

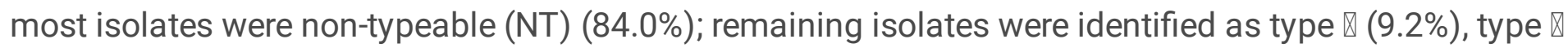
(3.4\%), type $\otimes(2.5 \%)$, and type $\otimes(0.8 \%)$. The results of SCCmec typing for MRSA isolates between with and without MRCoNS were similar (Fig. 2). We found 39 STs for MRSA isolates and the dominant types were ST5, ST45, ST59 and ST188. It was worth noted that most STs (including ST398, ST338, ST59, ST1281, ST188, ST88, ST2592, ST5, ST6, ST7, ST630 and ST45) were found in both MRSA isolates with and without MRCoNS.(Fig. 3).

\section{Discussion}

As the first study to determine epidemiology of MRSA nasal colonization for HIV-infected patients in mainland China, we found the prevalence $(11.89 \%)$ was higher than the result of a global meta-analysis which showed the pooled worldwide prevalence was 7\% (95\% $\mathrm{Cl} 5 \%-7 \%$ ) in adult HIV-infected patients [9]. Moreover, the prevalence in our study was also higher than other high-risk populations such as dialysis patients $(6.2 \%$; $95 \% \mathrm{Cl} 4.2 \%-8.5 \%)$ [15], men who have sex with men $(1.6 \% ; 95 \% \mathrm{Cl}$ : $0.5-2.6 \%)$ [16] and injection drug users (7.4\%) [17]. This high prevalence of MRSA colonization could be explained by high rates of antibiotic use and not effective infection control programs. Further studies need to confirm the reasons.

We found HIV-infected patients with diagnosis of respiratory tract infection in the previous 6 months were a more likely to have MRSA nasal colonization, which could be associated with the more frequently respiratory episode occur in HIV-infected population and bacteria colonize in the nasal cavity as the respiratory barrier weakens[18]. Male gender was a risk factor for MRSA colonization without MRCoNS, which was consistent with the previous results $[19,20]$. It may be related to the fact that $428 \mathrm{MSM}$ out of 843 men recruited for this study (data are not shown), study reported that MSM was found to have a high rate of MRSA infection in other contexts regardless of HIV status[21]. According to some studies, young people have higher risks of bacterial colonization [20,22], however, we did not find the same result in this study.

High proportions of resistance to penicillin, erythromycin, clindamycin and tetracycline were found in MRSA isolates, which is similar to observed studies [23-25]. This finding suggests medical professionals should pay more attention to the rational use of these antibiotics.

The detection rates of toxin genes for MRSA isolates in HIV-infected patients were lower than hospital patients [26-29], but similar to general populations [30-32]. Base on the results of SCCmec typing we found most MRSA isolates in this study were community-associated, which was similar to other studies $[33,34]$. However, hospital-associated SCCmec types were also found in this study, indicating a cross 
transmission between the community and hospital facilities. Moreover, the dominant STs (ST5, ST45, ST59 and ST188) in this study were consistent with other community-based populations [26,35-36], but some other STs were also previously reported in hospital patients (ST338 and ST1) [37-39] and animals ( ST398 and ST1) [40-42]. The MLST findings also indicated the cross transmission between communities and hospital settings.

Comparisons of characteristics, including antibiotic resistance and toxin genes as well as results of molecular typing, for MRSA isolates between with and without MRCoNS isolates were of no significant difference, suggesting the phenotypic and molecular characteristics of MRSA colonization were not affected by co-colonizing with MRCoNS.

To our knowledge, it is the first molecular epidemiological study of MRSA colonization with a large sample size among HIV patients in mainland China. However, some potential limitations also need to be considered. Firstly, we could not draw a cause conclusion for MRSA colonization in HIV patients because of the cross-sectional study design. Secondly, we administered the sampling at only one site, which may underestimate the prevalence of MRSA colonization. Thirdly, colonization was only assessed on the day of enrollment. Evaluation of durability of colonization may be useful to fully understand MRSA colonization dynamics.

\section{Conclusions}

HIV-infected patients remain a highly vulnerable population for MRSA colonization, and men and diagnosis of respiratory tract infections in the previous 6 months are risk factors. Risk of MRSA colonization were not affected by co-colonizing with MRCoNS due to the insignificant risk factor analysis and undifferentiated phenotypic and molecular characteristics between the two groups. Our findings emphasize regular surveillance of MRSA nasal colonization and the organization of preventive measures play an important role in controlling the potential transmission of MRSA in HIV-infected patients.

\section{Abbreviations}

MRSA: methicillin-resistant Staphylococcus aureus; MRCoNS: Methicillin-resistant coagulase-negative Staphylococci; pvl: Panton-Valentine leukocidin; tst: toxic shock syndrome toxin.

\section{Declarations}

\section{Acknowledgements}

Not applicable.

\section{Authors' contribution}


YZJ conceptualized the study. LY and LJL were responsible for data curation, formal analysis and wrote the original draft. LLH and CWP were responsible for resources and supervision. WYY and YJP were responsible for validation and visualization. HSP and ZWC were responsible for investigation and Laboratory operation. YZJ and YXH reviewed and edited the original draft.All authors read and approved the final manuscript.

\section{Funding}

This research was funded by Guangdong Science and Technology Plan Project (grant number 2014A020213013) and National Natural Science Foundation of China (grant number 81973069 , 81602901).

\section{Availability of data and materials}

The datasets used and/or analyzed during the current study are available from the corresponding author on reasonable request.

\section{Ethics approval and consent to participate}

This study was approved by the Ethics Committee of Guangdong Pharmaceutical University. II participants signed an informed consent.

\section{Consent for publication}

Not applicable.

\section{Competing interests}

The authors declare that they have no competing interests.

\section{References}

1. David MZ, Daum RS. Community-Associated Methicillin-Resistant Staphylococcus aureus: Epidemiology and Clinical Consequences of an Emerging Epidemic. Clin Microbiol Rev. 2010;23(3):616-87.

2. Hassoun A, Linden PK, Friedman B. Incidence, prevalence, and management of MRSA bacteremia across patient populations-a review of recent developments in MRSA management and treatment. Crit Care. 2017;21(1):211.

3. Sahreena L, Kunyan Z. Methicillin-Resistant Staphylococcus aureus: Molecular Characterization, Evolution, and Epidemiology. Clin Microbiol Rev. 2018;31(4): e00020-18.

4. Shorr AF, Tabak YP, Gupta V, Johannes RS, Liu LZ, Kollef MH. Morbidity and cost burden of methicillin-resistant Staphylococcus aureus in early onset ventilator-associated pneumonia. Crit Care. 2006;10(3):R97. 
5. Vidaur L, Planas K, Sierra R, Dimopoulos G, Ramirez A, Lisboa T, et al. Ventilator-associated pneumonia - Impact of organisms on clinical resolution and medical resources utilization. Chest. 2008;133:625-632.

6. Becker K, Heilmann C, Peters G. Coagulase-Negative Staphylococci. Clin Microbiol Rev. 2014;27(4):870-926.

7. Martins A, Cunha Mde L. Methicillin resistance in Staphylococcus aureus and coagulase-negative staphylococci: epidemiological and molecular aspects. Microbiol Immunol. 2007;51(9):787-795.

8. Al-Bakri AG, Al-Hadithi H, Kasabri V, Othman G, Kriegeskorte A, Becker K. The epidemiology and molecular characterization of methicillin-resistant staphylococci sampled from a healthy Jordanian population. Epidemiol Infect. 2013;141(11):1-8.

9. Sabbagh P, Riahi SM, Gamble HR, Rostami A. The global and regional prevalence, burden, and risk factors for methicillin-resistant Staphylococcus aureus colonization in HIV-infected people: A systematic review and meta-analysis. Am J Infect Control. 2019;47(3):323-333.

10. Wu CJ, Ko WC, Ho MW, Lin HH, Yang YL, Lin JN, et al. Prevalence of and risk factors for methicillinresistant Staphylococcus aureus colonization among human immunodeficient virus-infected outpatients in Taiwan: oral Candida colonization as a comparator. J Oral Microbiol. 2017;9(1):1322446.

11. McDonald LC, Lauderdale TL, Lo HJ, Tsai JJ, Hung CC. Colonization of HIV-infected outpatients in Taiwan with methicillin-resistant and methicillin-susceptible Staphylococcus aureus. Int J STD AIDS. 2003;14(7):473-477.

12. Mehrotra M, Wang G, Johnson WM. Multiplex PCR for detection of genes for Staphylococcus aureus enterotoxins, exfoliative toxins, toxic shock syndrome toxin 1 , and methicillin resistance. $\mathrm{J}$ Clin Microbiol. 2000;38(3):1032-1035.

13. Zhang K, Mcclure JA, Conly JM. Enhanced multiplex PCR assay for typing of staphylococcal cassette chromosome mec types I to $\mathrm{V}$ in methicillin-resistant Staphylococcus aureus. Mol Cell Probe. 2012;26(5):218-221.

14. Enright MC, Day NP, Davies CE, Peacock SJ, Spratt BG. Multilocus sequence typing for characterization of methicillin-resistant and methicillin-susceptible clones of Staphylococcus aureus. J Clin Microbiol. 2000;38(3):1008-1015.

15. Zacharioudakis IM, Zervou FN, Ziakas PD, Mylonakis E. Meta-analysis of methicillin-resistant Staphylococcus aureus colonization and risk of infection in dialysis patients. J Am Soc Nephrol. 2014;25(9):2131-2141.

16. Antoniou T, Devlin R, Gough K, Mulvey M, Katz KC, Zehtabchi M, et al. Prevalence of communityassociated methicillin-resistant Staphylococcus aureus colonization in men who have sex with men. Int J STD AIDS. 2009;20(3):180-183.

17. Al-Rawahi GN, Schreader AG, Porter SD, Roscoe DL, Gustafson R, Bryce EA. Methicillin-resistant Staphylococcus aureus nasal carriage among injection drug users: six years later. J Clin Microbiol. 2008;46(2):477-479. 
18. Miller R. HIV-associated respiratory diseases. 1996;348(9023):307-312.

19. Popovich KJ, Weinstein RA, Aroutcheva A, Rice T, Hota B. Community-Associated MethicillinResistant Staphylococcus aureus and HIV:Intersecting Epidemics. Clin Infect Dis. 2010;50(7):979987.

20. Furuno JP, Johnson JK, Schweizer ML, Uche A, Stine OC, Shurland SM, et al. Community-associated Methicillin-resistant Staphylococcus aureus Bacteremia and Endocarditis among HIV Patients: A cohort study. BMC Infect Dis. 2011;11:298.

21. Diep BA, Chambers HF, Graber CJ, Szumowski JD, Miller LG, Han LL, et al. Emergence of multidrugresistant, community-associated, methicillin-resistant Staphylococcus aureus clone USA300 in men who have sex with men. Ann. Intern. 2008;148(4):249-257.

22. Askarian M, Zeinalzadeh A, Japoni A, Alborzi A, Memish ZA. Prevalence of nasal carriage of methicillin-resistant Staphylococcus aureus and its antibiotic susceptibility pattern in healthcare workers at Namazi Hospital, Shiraz, Iran. Int J Infect Dis. 2009;13(5):e241-247.

23. Regina Pedrosa Soares C, de Lira CR, Cunha MAH, Romão de Souza Junior V, Lopes de Melo F, de Araújo PSR, et al. Prevalence of nasal colonization by methicillin-resistant Staphylococcus aureus in outpatients living with HIV/AIDS in a Referential Hospital of the Northeast of Brazil. BMC Res Notes. 2018;11(1):794.

24. Lemma MT, Zenebe Y, Tulu B, Mekonnen D, Mekonnen Z. Methicillin Resistant Staphylococcus aureus among HIV Infected Pediatric Patients in Northwest Ethiopia: Carriage Rates and Antibiotic Co-Resistance Profiles. PLOS ONE. 2015;10(9):e0137254.

25. Groome MJ, Albrich WC, Wadula J, Khoosal M, Madhi SA. Community-onset Staphylococcus aureus bacteraemia in hospitalised African children: high incidence in HIV-infected children and high prevalence of multidrug resistance. Paediatr Int Child Health. 2012;32(3):140-6.

26. Goudarzi M, Seyedjavadi SS, Nasiri MJ, Goudarzi H, Sajadi Nia R, Dabiri H. Molecular characteristics of methicillin-resistant Staphylococcus aureus (MRSA) strains isolated from patients with bacteremia based on MLST, SCCmec, spa, and agr locus types analysis. [23]Microb Pathog 2017;104:328-335.

27. Katsarou I, Paraskevopoulou NM, Papadimitriou-Olivgeris M, Giormezis N, Militsopoulou M, Kolonitsiou F, et al. Fatality of Staphylococcus aureus infections in a Greek university hospital: role of inappropriate empiric treatment, methicillin resistance, and toxin genes' presence. Eur $\mathrm{J}$ Clin Microbiol Infect Dis. 2020;39(3):443-450.

28. Jiménez JN, Ocampo AM, Vanegas JM, Rodríguez EA, Garcés CG, Patiño LA, et al. Characterisation of virulence genes in methicillin susceptible and resistant Staphylococcus aureus isolates from a paediatric population in a university hospital of Medellín, Colombia. Mem Inst Oswaldo Cruz. 2011;106(8):980-985.

29. Sila J, Sauer P, Kolar M. Comparison of the prevalence of genes coding for enterotoxins, exfoliatins, panton-valentine leukocidin and tsst- 1 between methicillin-resistant and methicillin-susceptible 
isolates of Staphylococcus aureus at the university hospital in olomouc. Biomed Pap Med Fac Univ Palacky Olomouc Czech Repub. 2009;153(3):215-218.

30. Mobasherizadeh S, Shojaei H, Azadi D, Havaei SA, Rostami S. Molecular characterization and genotyping of methicillin-resistant Staphylococcus aureus in nasal carriage of healthy Iranian children. J Med Microbiol. 2019;68(3):374-378.

31. Wu D, Li X, Yang Y, Zheng Y, Wang C, Deng L, et al. Superantigen gene profiles and presence of exfoliative toxin genes in community-acquired meticillin-resistant Staphylococcus aureus isolated from Chinese children. J Med Microbiol. 2011;60(Pt 1):35-45.

32. Xie X, Dai X, Ni L, Chen B, Luo Z, Yao Y, et al. Molecular epidemiology and virulence characteristics of Staphylococcus aureus nasal colonization in medical laboratory staff: comparison between microbiological and non-microbiological laboratories. BMC Infect Dis. 2018;18(1):122.

33. Ohadian Moghadam S, Modoodi Yaghooti M, Pourramezan N, Pourmand MR. Molecular characterization and antimicrobial susceptibility of the CA-MRSA isolated from healthcare workers, Tehran, Iran. Microb Pathog 2017;107:409-412.

34. Kim ES, Song JS, Lee HJ, Choe PG, Park KH, Cho JH. A survey of community-associated methicillinresistant Staphylococcus aureus in Korea. J Antimicrob Chemother 2007;60(5):1108-1114.

35. Lin J, Liang J, Zhang T, Bai C, Ye J, Yao Z. Dose-response associations of methicillin-resistant Staphylococcus aureus between school environmental contamination and nasal carriage by elementary students. Infect Drug Resist. 2018;11:773-782.

36. Lin J, Wu C, Ou Q, Lin D, Zhang T, Bai C, et al. Nasal colonization of Staphylococcus aureus colonal complex 5: Prevalence, influencing factors, and phenotypic and molecular characteristics in pregnant Chinese women. Am J Infect Control. 2017;45(10):1106-1110.

37. Lin J, Wu C, Yan C, Ou Q, Lin D, Zhou J, et al. A prospective cohort study of Staphylococcus aureus and methicillin-resistant Staphylococcus aureus carriage in neonates: the role of maternal carriage and phenotypic and molecular characteristics. Infect Drug Resist. 2018;11:555-565.

38. Tang YT, Cao R, Xiao N, Li ZS, Wang R, Zou JM, et al. Molecular epidemiology and antimicrobial susceptibility of methicillin-resistant, Staphylococcus aureus, isolates in Xiangyang, China. J Glob Antimicrob Resist. 2018;12:31-36.

39. Omuse G, Van Zyl KN, Hoek K, Abdulgader S, Kariuki S, Whitelaw A. Molecular characterization of Staphylococcus aureus isolates from various healthcare institutions in Nairobi, Kenya: a cross sectional study. Ann Clin Microbiol Antimicrob. 2016;15(1):51.

40. Conceição $T$, de Lencastre $H$, Aires-de-Sousa M. Frequent isolation of methicillin resistant Staphylococcus aureus (MRSA) ST398 among healthy pigs in Portugal. PLOS ONE. 2017;12(4):e0175340.

41. Schauer B, Krametter-Frötscher R, Knauer F, Ehricht R, Monecke S, Feßler AT, et al. Diversity of methicillin-resistant Staphylococcus aureus (MRSA) isolated from Austrian ruminants and New World camelids. Vet Microbiol. 2018;215:77-82. 
42. Wipf JR, Perreten V. Methicillin-resistant Staphylococcus aureus isolated from dogs and cats in Switzerland. Schweiz Arch Tierheilkd. 2016;158(6):443-450.

Figures

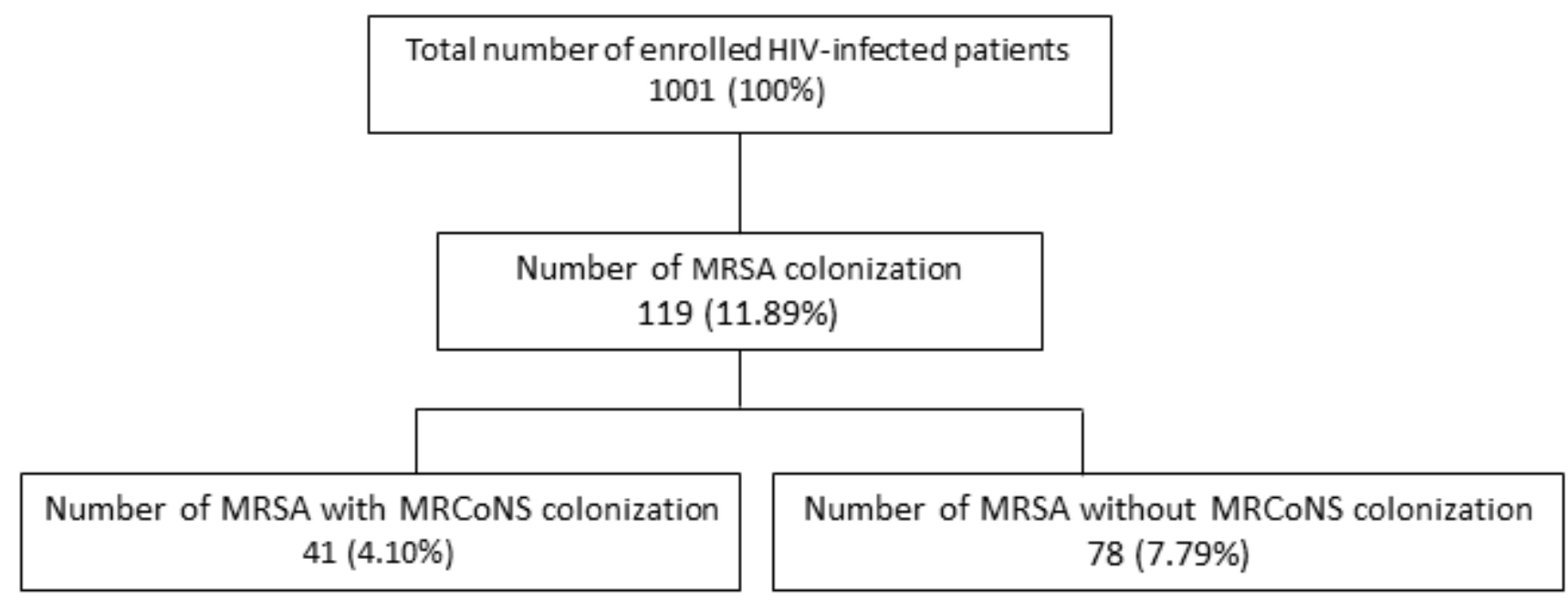

\section{Figure 1}

Enrollment and prevalence of MRSA colonization. MRSA, methicillin-resistant Staphylococcus aureus; MRCoNS, methicillin-resistant coagulase-negative Staphylococci. 


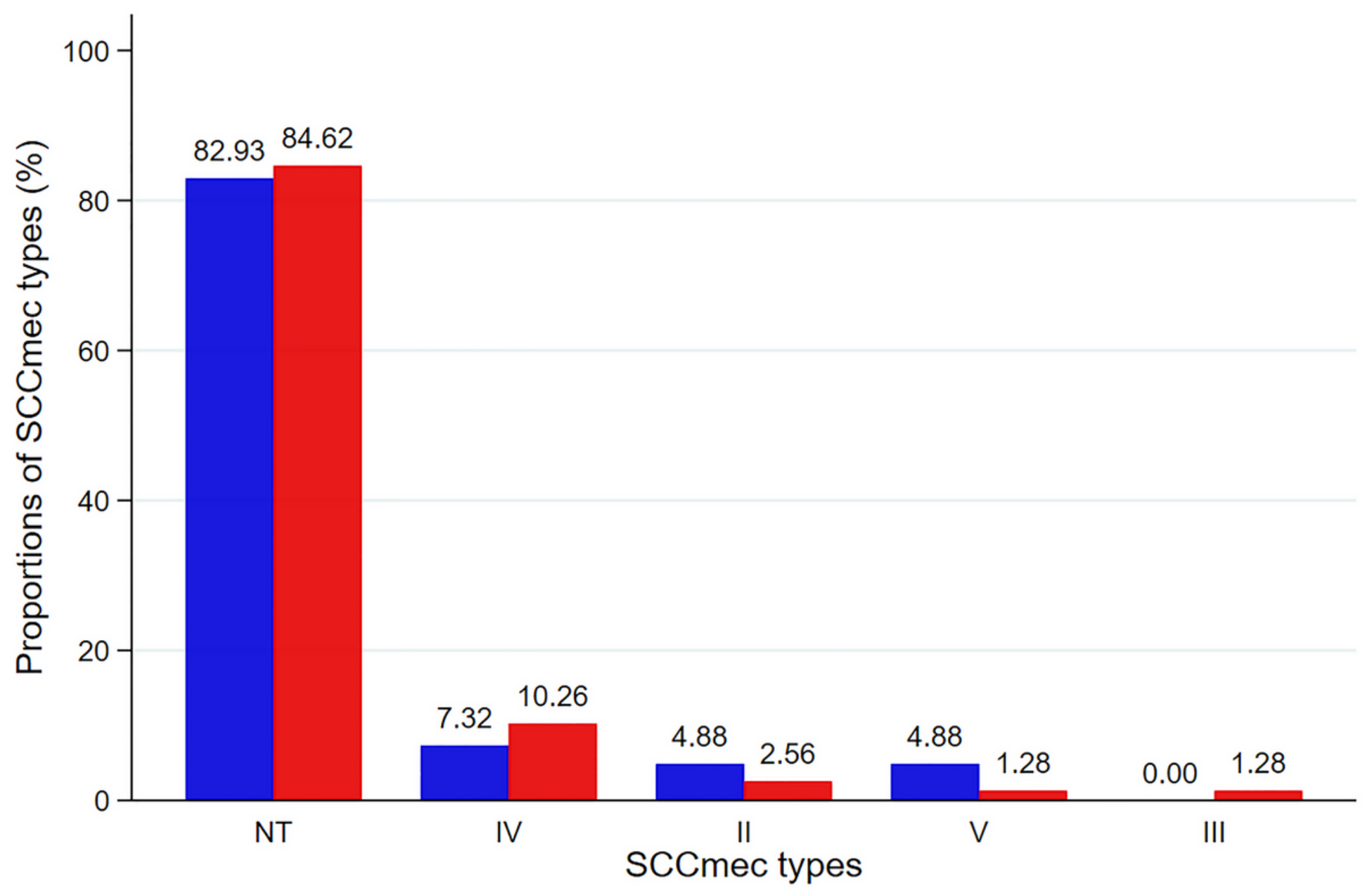

MRSA with MRCoNS

MRSA without MRCoNS

\section{Figure 2}

Distribution of SCCmec types for MRSA isolates with and without MRCoNS isolates. MRSA, methicillinresistant Staphylococcus aureus; MRCoNS, methicillin-resistant coagulase-negative Staphylococci; SCCmec, staphylococcal cassette chromosome mec; NT, non-typeable. 


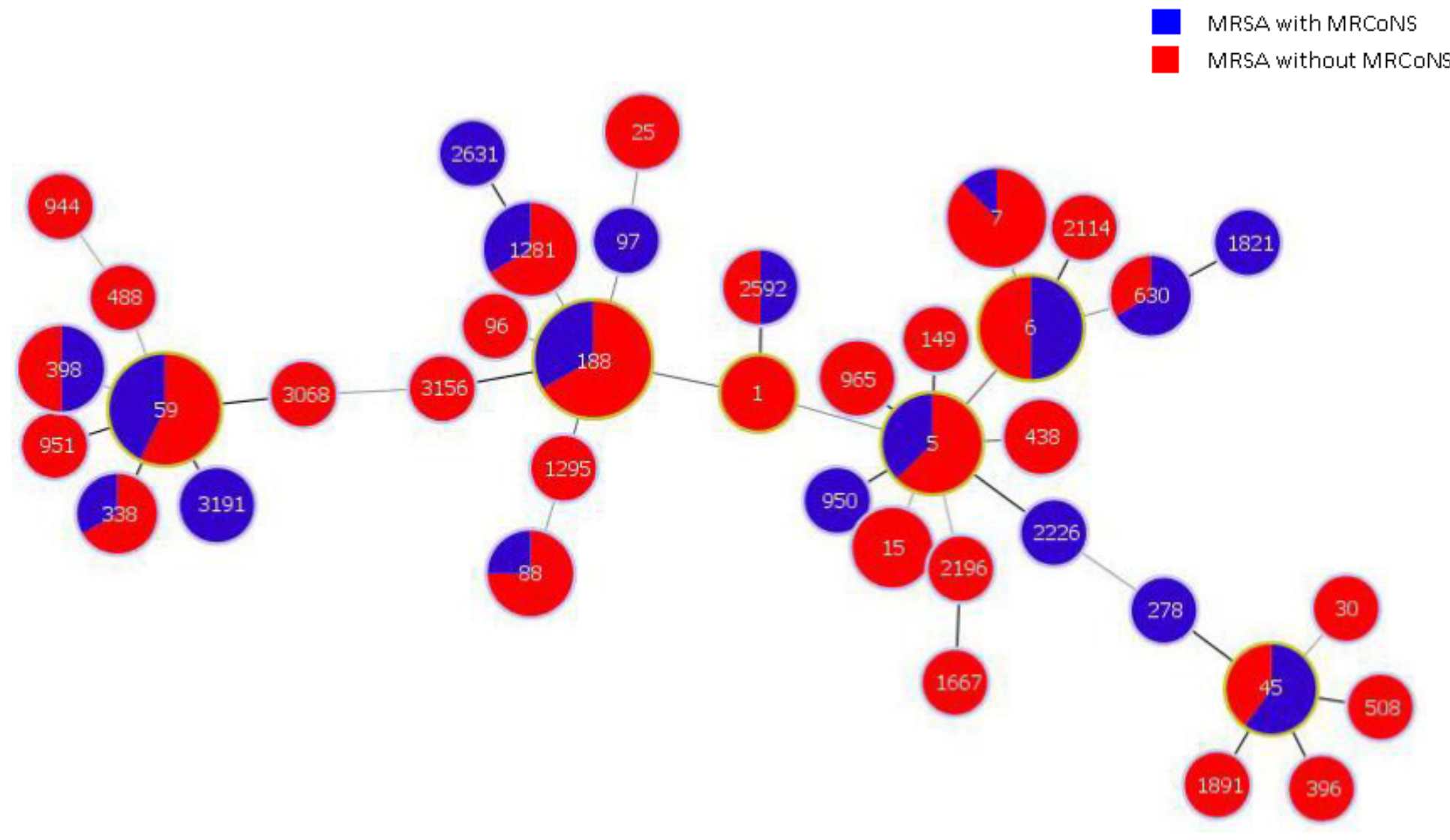

Figure 3

The sequence type relationship of MRSA isolates between with and without MRCoNS isolates. MRSA, methicillin-resistant Staphylococcus aureus; MRCoNS, methicillin-resistant coagulase-negative Staphylococci. 